\title{
集束化护理预防血液透析患者导管相关血流感染的 效果观察
}

\author{
王晓燕 ${ }^{*}$ 张 沛 刘梦怡 刘 婕 席红梅 \\ 陕西省渭南市中心医院，陕西 714000
}

摘 要: 目的: 研究集束化护理预防血液透析患者导管相关血流感染的效果。方法：从到我院就医的血液透析患 者中选择110例, 平均分为对照组和观察组, 每组55例, 分别给予常规护理和集束化护理, 比较两组患者血液透析导 管相关血流感染率的发生情况，心理状态以及护理满意度。结果：护理后观察组导管相关血流感染发生率为 $1.82 \%$, 显著低于对照组 $10.91 \%(P<0.05)$; 护理后观察组SDS与SAS评分均优于对照组 $(P<0.05)$; 护理后观察组护理满 意度为 $96.36 \%$, 显著高于对照组 $85.45 \%(P<0.05)$ 。结论：对血液透析患者给予集束化护理千预, 能够有效预防导 管相关性血流感染疾病，值得在临床上推广应用。

关键词：集束化护理；血液透析；导管相关性血流感染；护理满意度

\section{Effect of Cluster Nursing on Prevention of Catheter-related Bloodstream Infection in Hemodialysis Patients}

\author{
Xiao-Yan Wang*, Pei Zhang, Meng-Yi Liu, Jie Liu, Hong-Mei Xi \\ Weinan Central Hospital, Weinan 714000, Shaanxi, China
}

\begin{abstract}
Objective: To study the effect of cluster nursing on prevention of catheter-related bloodstream infection in hemodialysis patients. Method: 110 hemodialysis patients were selected from our hospital and divided into control group and observation group with 55 cases in each group. Routine nursing and cluster nursing were given respectively. The incidence of hemodialysis catheter-related bloodstream infection rate, psychological status and nursing satisfaction of the two groups were compared. Result: After nursing, the incidence of catheter-related bloodstream infection in the observation group was $1.82 \%$, significantly lower than $10.91 \%$ of the control group $(P<0.05)$; the SDS and SAS scores of the observation group were better than those of the control group $(P<0.05)$; the nursing satisfaction of the observation group was $96.36 \%$, significantly higher than that of the control group $85.45 \%(P<0.05)$. Conclusion: Cluster nursing intervention for hemodialysis patients can effectively prevent catheter-related bloodstream infection, which is worthy of clinical application.
\end{abstract}

Keywords: Cluster nursing; hemodialysis; catheter related bloodstream infection; nursing satisfaction

\section{一、前言}

血液透析是肾脏衰竭临床上主要治疗方法, 可建立和维持有效血管通路, 保证血液透析可以顺利完成。在临床上 的实际操作中, 往往会留置血液透析导管来进行治疗, 能够有效缓解患者疼痛感, 但是相应的缺陷在于存在更大的概 率发生导管相关血流感染，进而会产生菌血症，降低血液透析治疗效果，甚至还会威胁患者的生命安全 ${ }^{[1]}$ 。因此血液 透析患者临床治疗过程中, 还需要给予专业的护理干预。就临床数据来看, 常规护理干预方法依然会存在较高的并发 症发生率，相比来讲集束化护理作为一种相对新型的干预方法，通过对各环节的优化改善，可以进一步降低并发症发 生率，提高血液透析临床治疗效果。为对集束化护理干预临床应用效果进行研究，本次选择了在本院接受治疗的 110 例血液透析患者来参与观察。以下为报道内容。

*通讯作者: 王晓燕, 1972年5月，女，汉族，陕西渭南人，现任陕西省渭南市中心医院主任护师，本科。研究方 向：内科护理。 


\section{二、资料与方法}

(一) 一般资料

从到我院就医的血压透析患者中随机选择 110 例来参与本次研究, 对其历史资料做回顾性研究。所有患者被平 均分为对照组和观察组, 每组患者各为55例, 且经过比较两组患者一般资料无明显差异, 可以用于统计学分析 $(P>$ $0.05)$ 。对照组内男女患者的比例为 $35: 20$, 最小/最大年龄为 52 岁和 73 岁, 平均年龄 $(61.25 \pm 3.26$ ) 岁, 其中包括 26 例 糖尿病肾病患者、18例急性肾衰竭患者与11例慢性肾小球肾炎患者, 共有22例右股静脉置管和33例右颈内静脉置管患 者。观察组内男女患者的比例为 $28: 27$, 最小/最大年龄为 54 岁和 76 岁, 平均年龄 $(61.48 \pm 3.41)$ 岁, 其中包括 23 例糖尿 病肾病患者、17例急性肾衰竭患者与15例慢性肾小球肾炎患者，共有26例右股静脉置管和29例右颈内静脉置管患者。

1. 纳人标准

(1) 接受血液透析治疗时间超过 3 个月，每周透析次数在 2 次以上的患者。

(2) 对本次研究知情并自愿参与且配合度高的患者。

(3) 病情稳定且生存期在 6 个月以上的患者 ${ }^{[2]}$ 。

2. 排除标准

（1）伴有肺部重度感染、心力衰竭等严重并发症的患者。

(2) 中途退出观察研究的患者。

3. 导管相关感染诊断标准

（1）患者1次或多次血培养中培养出一种已识别的病原体，以及该种病原体与其他部位感染没有关系.

（2）患者体温达到 $38.0^{\circ} \mathrm{C}$ 以上, 伴有寒战或低血压临床症状, 且病原体与其他部位感染没有关系; 或者是微生物 为较常见共生物, 且在2次及以上不同批次抽血的血培养中均存在该种微生物 ${ }^{[3]}$ 。

(二) 方法

对照组患者给予常规护理干预, 对于人院患者要给予专业指导, 完成相关置管以及穿刺等流程操作, 并且向患者及 家属讲解血液透析的作用, 告知其各项注意内容。在患者血液透析治疗的过程中, 需要做好各项生命体征的严密监测。

观察组患者给予集束化护理干预，主要包括以下几个方面:

1. 医护人员培训

对医护人员进行定期的专业培训, 开展血液透析治疗过程风险课程, 讲解操作流程, 并总结上一阶段工作确定各 注意事项, 且需要在课程结束后进行实战演练, 提高所有医护人员的专业技能水平, 可以专业且熟练的完成血液透析 治疗操作, 掌握患者病情并及时就出现的异常做有效处理, 保证患者治疗过程的安全性, 且预防并发症的发生 ${ }^{[4]}$ 。

\section{2. 物品器械管理}

对透析室内的所有物品器械必须严格管理, 定期做好室内空气消毒, 在进人透析室内后均需要采取无菌操作, 减 少人员流动，降低患者感染情况的发生概率。

\section{3. 健康教育指导}

护理人员要注意患者心理状态的变化, 与其建立良好的沟通渠道, 通过有效交流掌握患者基本情况, 并根据其文 化程度来选择合适的方法给予健康教育。讲解疾病发病原因、临床症状、治疗方法和注意事项, 并且要告知其血液透 析治疗可能会产生的并发症，并强调各项预防措施的重要性，提高其配合度。

\section{4. 手部卫生管理}

在患者进行血液透析治疗时, 医护人员需要严格按照专业原则做好手部卫生管理, 保证接触敷料以及导管的所有 环节均不会存在感染风险。可选用乙醇擦手液对手部做全面清洗消毒, 安排专门的质控员对卫生情况进行检查评价, 还要定期询问各阶层人员的意见与建议, 对上一阶段工作中存在的问题作出调整改善 ${ }^{[5]}$ 。整个治疗操作流程, 所有医 护人员均需要佩戴口罩帽子以及手套，严格落实无菌屏障标准。

\section{5. 穿刺与感染监测}

优先选择右颈内静脉为穿刺部位, 部分特殊情况下可以选择右股静脉穿刺。穿刺前需要利用含氯已定进行有效消 毒, 透析治疗后要每天监测患者体温变化, 观察穿刺部位是否出现疼痛红肿等症状, 且要做好详细记录。如果发现任 何异常, 护理人员要及时告知主治医师, 及时采取有效措施处理。一般情况下, 在患者透析结束后的 $1 \mathrm{~h}$ 内要注意观察 
患者是否存在发热畏寒症状, 对导管进行检查确认是否滋生细菌 ${ }^{\left[{ }^{6}\right.}$ 。护理人员要定期对患者更换敷料并消毒, 预防穿 刺部位发生感染。

6. 敷料与导管护理

要利用无菌纱布和透明敷料对插管部位进行覆盖，如果敷料遇水、遇污渍以及松动脱落等情况发生，护理人员必 须立刻进行更换, 正常情况下需要在穿刺后的第二天对敷料进行更换, 然后根据患者病情恢复状态调整更换时间, 一 般为 $3 \mathrm{~d} / 1$ 次的频率 ${ }^{[7]}$ 。另外, 要注意导管的预防感染护理, 在使用导管前要利用碘酒对局部皮肤做消毒处理, 并将封 管内残留的血凝块和毒素清除干净, 确保管腔保持通畅。待血液透析结束好依然需要对导管做全面消毒处理, 并向内 注人肝素盐水封管，盖上肝素帽，最后包裹固定即可。

(三) 观察指标与评价标准

1. 治疗 3 个月后，观察两组患者导管相关血流感染的发生情况。

2. 应用焦虑自评量表 (SAS) 与抑郁自评量表 (SDS) 对两组患者的心理状态进行评分, 得分越高表示患者心理 状态越不乐观。

3. 应用医院自制的调查问卷, 统计两组患者的护理满意度情况, 分为满意、基本满意与不满意, 满意度 $=$ 满意 率+基本满意率。

( 四) 统计学方法

文章数据用SPSS19.0软件处理，计量资料采用 $\mathrm{T}$ 检验，计数资料以 $x^{2}$ 检验。若 $P<0.05$, 则有统计学意义。

\section{三、结果}

(一) 组间比较导管相关血流感染发生情况

护理后观察组患者导管相关血流感染发生率为 $1.82 \% （ 1 / 55)$ ，显著低于对照组 $10.91 \% （ 6 / 55 ）$, 组间比较具有 统计学意义 $(P<0.05)$ 。

(二) 组间比较患者SAS与SDS评分情况

护理前两组患者心理状态评分无差异，护理后观察组SDS与SAS评分均显著优于对照组 $(P<0.05)$ 。见下表1。

表1 组间比较患者SAS与SDS评分 $(\bar{x} \pm s$, 分)

\begin{tabular}{cccccc}
\hline \multirow{2}{*}{ 组别 } & 例数 & \multicolumn{2}{c}{ SAS } & \multicolumn{2}{c}{ SDS } \\
\cline { 3 - 6 } & & 护理前 & 护理后 & 护理前 & 护理后 \\
\hline 对照组 & 55 & $70.86 \pm 6.13$ & $55.89 \pm 5.01$ & $67.98 \pm 6.08$ & $47.26 \pm 3.57$ \\
观察组 & 55 & $71.23 \pm 6.31$ & $34.26 \pm 4.15$ & $68.16 \pm 6.33$ & $35.94 \pm 4.15$ \\
$t$ & & 0.483 & 9.342 & 0.068 & 8.174 \\
$P$ & & 0.314 & 0.000 & 0.484 & 0.000 \\
\hline
\end{tabular}

(三) 组间比较患者护理满意度情况

护理后观察组护理满意度为 $96.36 \% ，$ 显著高于对照组 $85.45 \% （ P<0.05)$ 。见下表2。

表2 组间比较患者护理满意度情况 $[\boldsymbol{n}(\%)]$

\begin{tabular}{cccccc}
\hline 组别 & 例数 & 满意 & 基本满意 & 不满意 & 满意度 \\
\hline 对照组 & 55 & $27(49.09)$ & $20(36.36)$ & $8(14.55)$ & $47(85.45)$ \\
观察组 & 55 & $35(63.64)$ & $18(32.73)$ & $2(3.64)$ & $53(96.36)$ \\
$x^{2}$ & & & & 8.261 \\
$P$ & & & & 0.026 \\
\hline
\end{tabular}

\section{四、讨论}

肾衰竭在临床上多是采用血液透析的方法治疗，临床效果比较理想，对于促进患者病情改善，并维持患者的生命 具有重要意义。但是血液透析是一个长期治疗的过程, 由此使得患者在治疗过程中可能出现并发性疾病, 进而影响到 治疗效果。因此必须注意预防导管相关性血流感染并发症的发生, 以免降低治疗效果, 确保患者生命安全。相比常规 护理干预方法, 集束化护理干预的预防感染效果更佳, 通过本次观察研究可知, 观察组患者给予集束化护理干预, 在 导管相关性血流感染发生率、患者SAS与SDS评分以及护理满意度各方面，均显著优于对照组 $(P<0.05)$ 。 
综上所述, 对血液透析患者实施集束化护理干预, 能够进一步预防导管相关性血流感染的发生, 获得更高治疗有 效率，值得在临床上推广应用。

\section{参考文献:}

[1]胡云华.集束化护理预防血液透析患者导管相关血流感染的效果评价[J]. 河北医药, 2020,42(09):1434-1436+1440.

[2]曾水珍.集束化护理对血液透析患者导管相关性血流感染的预防效果观察[J].中外医学研究, 2019,17(09):72-73.

[3]王丽. 集束化护理预防血液透析导管相关血流感染及改善患者负性心理状态的效果分析 [J]. 基层医学论坛, 2019,23(06):760-763.

[4]陈月梅.集束化护理在预防血液透析导管相关血流感染的效果观察[J].实用临床护理学电子杂志, 2019,4(05):17.

[5]平立静.对进行血液透析的患者实施集束化护理对预防其发生导管相关血流感染的效果 [J]. 当代医药论丛, 2019,17(02):279-280.

[6]熊素华. 集束化护理千预在预防血液透析导管相关血流感染的效果观察[J]. 实用临床护理学电子杂志, 2018,3(06):49.

[7]吴霞红.集束化护理在预防血液透析导管相关血流感染的效果观察[J].实用临床护理学电子杂志, 2017,2(51):53+58. 